\title{
Second-line chemotherapy for patients with advanced gastric cancer
}

\author{
Daisuke Takahari ${ }^{1}[$
}

Received: 2 December 2016/ Accepted: 22 February 2017/Published online: 4 March 2017

(c) The International Gastric Cancer Association and The Japanese Gastric Cancer Association 2017

\begin{abstract}
The first choice for treating patients with metastatic gastric cancer is chemotherapy, and combination therapy with fluorouracil, platinum, and trastuzumab has been established as the standard first-line chemotherapy. For further improvement of treatment outcomes, it is important to develop second- and third-line chemotherapy. In the first decade of this century, irinotecan and taxanes, cytotoxic agents, and various molecular targeted agents began to be developed as second-line therapy. Treatment with paclitaxcel weekly in combination with ramucirumab targeting vascular endothelial growth factor receptor 2 has become the first choice for second-line therapy. Immune checkpoint inhibitors are now being developed, and the current treatment strategies for advanced gastric cancer may undergo major changes in the future. This review summarizes the transitions and future prospects of clinical developments for second-line therapy in patients with advanced gastric cancer.
\end{abstract}

Keywords Gastric cancer · Paclitaxel · Irinotecan · Ramucirumab · Chemotherapy

\section{Introduction}

According to Torre at al. [1] gastric cancer was the fourth most frequently diagnosed cancer and the third leading cause of cancer-related death worldwide in 2012, with

Daisuke Takahari

daisuke.takahari@jfcr.or.jp

1 Department of Gastroenterology, Cancer Institute Hospital, Japanese Foundation for Cancer Research, 3-8-31, Ariake, Koto-ku, Tokyo 135-8550, Japan
951,600 new cases and 723,100 deaths globally. The prevalence of gastric cancer, though high on a global basis, is highest in East Asia. Treatment options for gastric cancer, such as surgery, chemotherapy, and radiotherapy, differ depending on the tumor status. Chemotherapy is the first choice for the treatment of patients with metastatic gastric cancer. In these patients, the main objectives of chemotherapy are survival prolongation, symptom palliation, and quality-of-life improvement. In the 1990s, prospective clinical trials and meta-analyses demonstrated the survival benefits of chemotherapy as compared with best supportive care (BSC) [2-5]. Clinical developments for first-line treatment of advanced gastric cancer (AGC) began to be increasingly actively developed in Asia, Europe, and the USA to enhance their clinical benefits. As a result, combination therapy with 5-fluorouracil (5-FU) plus platinum and with 5-FU plus platinum plus docetaxel/ epirubicin has been established as standard treatment [6-12].

The pooled analysis data from two phase III trials (JCOG9205 and JCOG9912) of first-line chemotherapy for patients with AGC conducted by the Japan Clinical Oncology Group confirmed that patients treated in the JCOG9912 trial had a better prognosis than patients in the JCOG9205 trial. This result can be interpreted as suggesting that the improved efficacy of first-line chemotherapy with 5-FU-based therapy and second-line chemotherapy as well as subsequent-line chemotherapy with new agents, such as irinotecan, docetaxel, and paclitaxel, contributed significantly to the improved survival [13]. In a phase III trial (SPIRITS) of first-line chemotherapy conducted to verify the superiority of S-1 and cisplatin (SP) over S-1, median overall survival (OS) was 13.0 months in the SP group and 11.0 months in the S-1 group [hazard ratio (HR) 0.77 ; $95 \%$ confidence interval 
(CI) $0.61-0.98 ; P=0.04]$. Seventy-five percent of the patients received second-line treatment after completion of the trial: those who received taxane (docetaxel or paclitaxel)-based posttreatment accounted for $51 \%$ of the SP group and $40 \%$ of the S-1 group, and those who received irinotecan-based posttreatment accounted for $14 \%$ and $15 \%$ respectively. From the results of these historical studies, a treatment strategy for first-line chemotherapy as well as for second- and subsequent-line chemotherapy is thought to be important for the prolongation of survival in AGC patients.

\section{Cytotoxic agents}

Data have been reported from a number of phase II and phase III trials conducted to investigate the efficacy of second-line chemotherapy with irinotecan, docetaxel, or paclitaxel in patients with AGC [14-33] (Table 1).

\section{Irinotecan and taxanes (docetaxcel, paclitaxcel, nab-paclitaxcel)}

The Arbeitsgemeinschaft Internistische Onkologie (AIO) in Germany conducted a phase III trial to verify the superiority of irinotecan over BSC in terms of OS with the aim of proving the efficacy of second-line chemotherapy for AGC [28]. Despite its initial plan to include a total of 120 patients in both treatment groups, the trial could not enroll more than 40 patients and had to be terminated prematurely. The median OS was 4.0 months in the irinotecan group $\left(250 \mathrm{mg} / \mathrm{m}^{2}\right.$ every 3 weeks $)$ and 2.4 months in the BSC group (HR 0.48; 95\% CI 0.25-0.92; $P=0.012$ ), suggesting the superiority of treatment in the irinotecan group. This was the first clinical trial, albeit in a small number of patients, that suggested the efficacy of second-line chemotherapy for AGC.

In Korea, a phase III trial was conducted to verify the superiority of docetaxel or irinotecan over BSC in terms of OS in second-line chemotherapy for AGC [29]. Patients were randomized into either a salvage chemotherapy (SLC) group or a BSC group at a ratio of 2:1 (133 patients in the SLC group and 69 patients in the BSC group). Those in the SLC group received either of the following treatments at the discretion of the investigator: docetaxel at $60 \mathrm{mg} / \mathrm{m}^{2}$ every 3 weeks or irinotecan at $150 \mathrm{mg} / \mathrm{m}^{2}$ every 2 weeks. The median OS was 5.3 months in the SLC group and 3.8 months in the BSC group, showing that survival in the SLC group was significantly prolonged (HR $0.657 ; 95 \%$ CI $0485-0.891 ; P=0.007)$. There was no statistically significant difference in OS between the docetaxel cohort (5.2 months) and the irinotecan cohort (6.5 months)
$(P=0.116)$. The incidence of adverse events was similar in both the docetaxel cohort and the irinotecan cohort (neutropenia, $15 \%$ vs $18 \%$; anemia, $30 \%$ vs $32 \%$ ). This was the first large-scale phase III trial that verified the efficacy of second-line chemotherapy in patients with AGC.

Another phase III trial, COUGAR-02, was conducted in patients with esophagogastric adenocarcinoma to compare docetaxel and active symptom control [30]. This was also the first phase III trial of second-line chemotherapy that evaluated health-related quality of life in patients previously treated with 5-FU and platinum. One hundred sixtyeight patients ( 84 for each group) were enrolled in the United Kingdom. The OS was 5.2 months in the docetaxel group $\left(75 \mathrm{mg} / \mathrm{m}^{2}\right.$ every 3 weeks; treatment was continued for up to 6 cycles), which was significantly longer than the 3.6 months in the active symptom control group (HR 0.67; 95\% CI $0.49-0.92 ; P=0.01)$. In the evaluation based on the EORTC Quality of Life Questionnaire-Core 30 (QLQ$\mathrm{C} 30$ ), although there was no significant difference in functional scales or overall quality of life between the two groups, assessments of some of the symptom subscales showed significantly better results in the docetaxel group compared with the active symptom control group (pain, $P=0.0008$; abdominal pain, $P=0.01$; nausea and vomiting, $P=0.02$; and constipation, $P=0.02$ ).

Paclitaxcel is an established standard treatment for a wide variety of cancer types. For the treatment of AGC, paclitaxcel was developed as an agent administered in a every 3 weeks regimen. Although it also shows a modest overall response rate (ORR) (26-27\%) as second-line chemotherapy, paclitaxcel is known to cause hematotoxicity, in particular, a high incidence (37-88\%) of grade 3 or greater neutropenia $[17,18]$. In Japan, a weekly regimen of paclitaxcel was actively developed to reduce hematotoxicity [19-21, 23, 24]. Because its efficacy is comparable to the every 3 weeks regimen while causing fewer severe adverse events, the weekly regimen of paclitaxcel has come to be widely used in clinical practice in Japan.

WJOG4007, a phase III trial that enrolled 223 patients in Japan, was conducted to verify the superiority of irinotecan over a weekly regimen of paclitaxcel [31]. The OS was 8.4 months in the irinotecan group $\left(150 \mathrm{mg} / \mathrm{m}^{2}\right.$ on days 1 and 15 , every 4 weeks) and 9.5 months in the group that received paclitaxcel weekly $\left(80 \mathrm{mg} / \mathrm{m}^{2}\right.$ on days 1,8 , and 15 , every 4 weeks), failing to demonstrate the superiority of irinotecan over a weekly regimen of paclitaxcel (HR 1.13 ; 95\% CI $0.86-1.49 ; P=0.38)$. Progression-free survival (PFS) was 2.3 months in the irinotecan group and 3.6 months in the group that received paclitaxcel weekly (HR 1.14; 95\% CI 0.88-1.49; $P=0.33$ ). The following grade 3 or higher adverse events were reported in the group that received paclitaxcel weekly and the irinotecan group: 


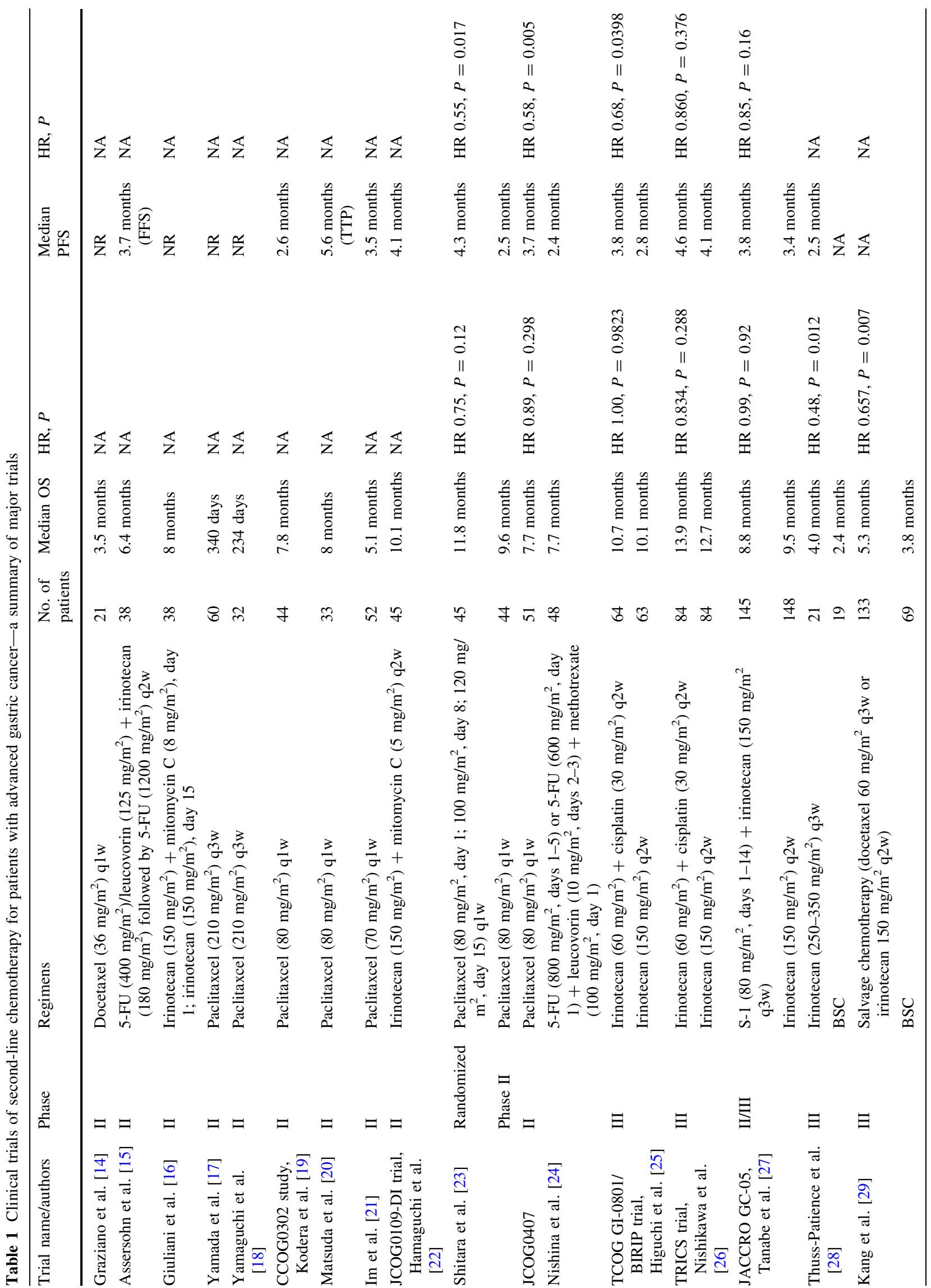




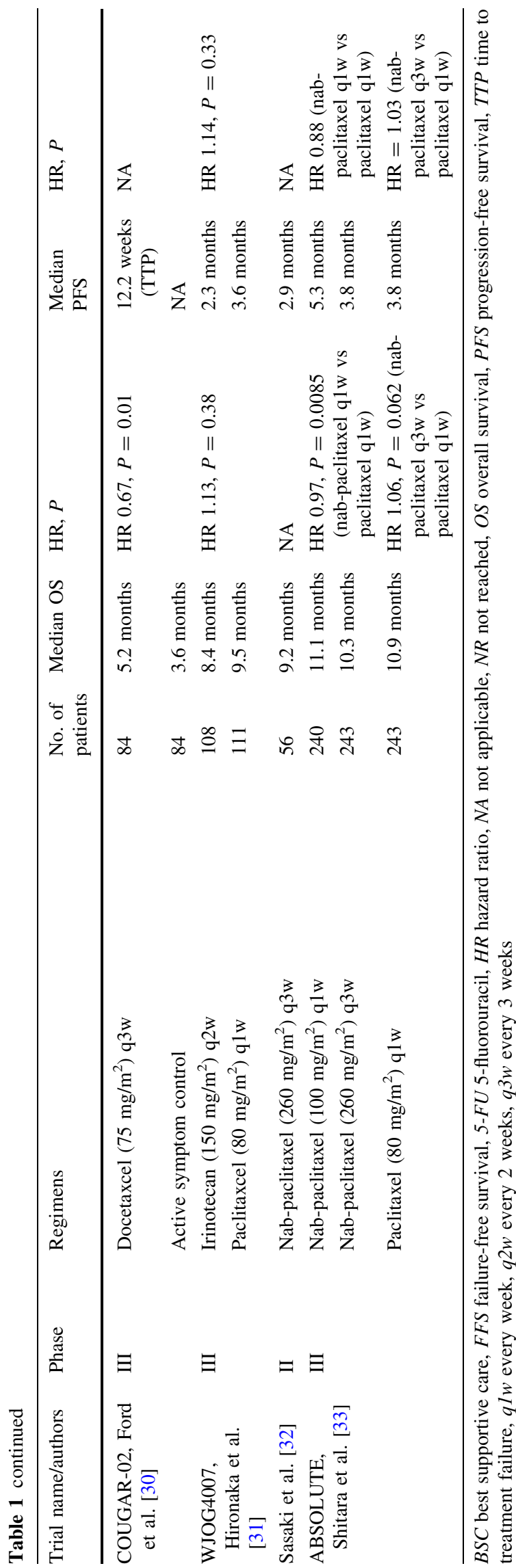

leukopenia $(20.4 \%$ and $19.1 \%$ respectively), neutropenia (28.7\% and $39.1 \%$ respectively), hemoglobin $(21.3 \%$ and $30.0 \%$ respectively), peripheral sensory neuropathy $(7.4 \%$ and $0 \%$ respectively), and febrile neutropenia (2.8\% and $9.1 \%$ respectively). Compared with the results of the two previously mentioned phase III trials (the AIO trial and the Korean trial) $[28,29]$, the OS tended to be longer in both the group that received paclitaxcel weekly and the irinotecan group. These results were considered to reflect the following facts: (1) patients assessed as having performance status 0 or 1 accounted for about $96 \%$; (2) about half of the patients had a single metastatic site; and (3) the proportion of patients with severe peritoneal metastasis was lower than that previously reported data (AIO trial, 43\%; Korean trial, $45 \%$; WJOG4007 trial, 25.6\%). On the basis of the results of the WJOG4007 trial, weekly paclitaxcel has become preferentially selected over irinotecan as second-line chemotherapy for AGC.

Nab-paclitaxcel, a 130-nm, albumin-bound nanoparticle formulation of paclitaxcel, does not contain Cremophor ${ }^{\mathbb{B}}$ EL or anhydrous ethanol, solvents present in conventional paclitaxcel formulations, and does not require patients to receive prior treatment for the prevention of hypersensitivity. In Japan, a phase II trial of nab-paclitaxcel given every 3 weeks ( $260 \mathrm{mg} / \mathrm{m}^{2}$ every 3 weeks) was conducted as second-line chemotherapy for patients with AGC. The ORR was $27.8 \%$, the median PFS was 2.9 months $(95 \%$ CI 2.4-3.6 months), and the median OS was 9.2 months (95\% CI 6.9-11.4 months) [32]. On the basis of the results of this phase II trial, nab-paclitaxcel given every 3 weeks was approved in Japan for the indication of AGC in February 2013. However, because paclitaxcel used globally was given as a weekly regimen, development of nab-paclitaxcel as a weekly regimen was highly demanded. Given this situation, ABSOLUTE, a phase III trial that enrolled 741 patients in Japan, was conducted [33]. The OS was 11.1 months in the group that received nab-paclitaxcel every week (100 mg/m ${ }^{2}$; days 1,8 , and 15 ; every 4 weeks) and 10.9 months in the control group, which received paclitaxcel every week (HR 0.97; 95\% CI 0.76-1.23; $P=0.0085$ ), demonstrating the noninferiority of weekly nab-paclitaxcel in comparison with weekly paclitaxcel. Meanwhile, the OS in the group that received nab-paclitaxcel every 3 weeks was 10.3 months (HR 1.06; 95\% CI $0.87-1.31 ; P=0.062$ ), failing to demonstrate noninferiority with respect to weekly paclitaxcel. Although the common adverse events reported in the group that received nab-paclitaxcel weekly were hematologic events, such as neutropenia, similar to those reported in the group that received paclitaxcel weekly, the incidence of peripheral sensory neuropathy in the group that received nab-paclitaxcel weekly was comparable to that in the group that received paclitaxcel weekly, and anaphylaxis, an event of 


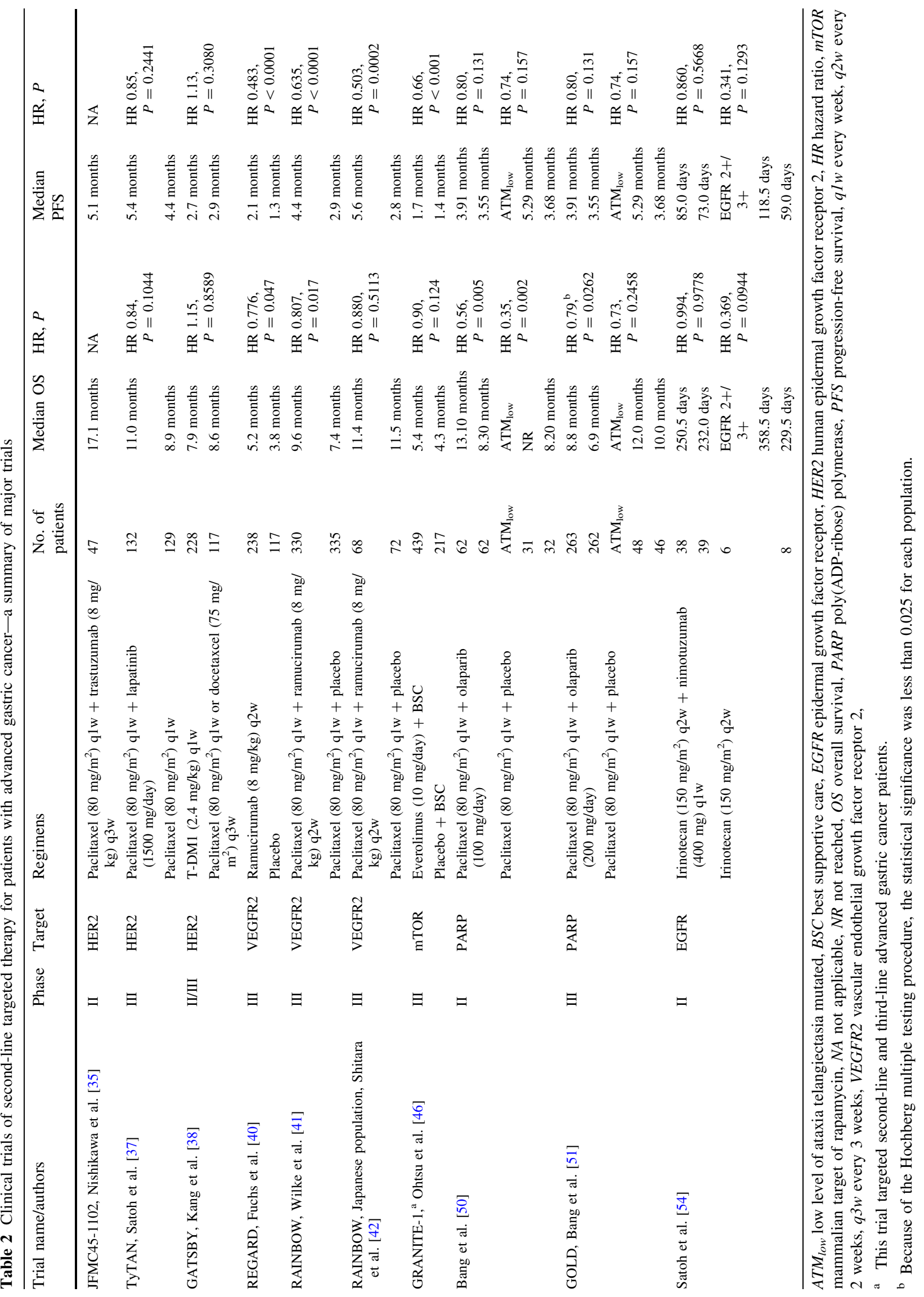


concern in the group that received paclitaxcel weekly (2\%), did not occur in any patients in the group that received nabpaclitaxcel weekly. If weekly nab-paclitaxcel were to be approved in Japan, it would become a treatment option as second-line chemotherapy for AGC.

\section{Molecular targeted therapies}

For the treatment of AGC, molecular targeted agents started to be developed in the first decade of this century, targeting various molecules. At present, trastuzumab, an anti-human epidermal growth factor receptor 2 (HER2) monoclonal antibody, and ramucirumab, an anti-vascular endothelial growth factor receptor 2 monoclonal antibody, are the only molecular targeted agents that have been successfully developed and are currently available on the market (Table 2). Several types of molecular targeted agents are currently being developed, and are expected to serve as future treatment options (Table 3).

\section{Anti-HER2 antibody}

Treatment with trastuzumab, an anti-HER2 monoclonal antibody, was established as a standard first-line therapy for AGC after its efficacy as first-line chemotherapy had been verified in HER2-positive patients [34]. No phase III trials have been conducted to investigate the efficacy and safety of trastuzumab as second-line therapy. In a phase II trial in HER2-positive patients with AGC who had previously been treated with chemotherapy other than trastuzumab therapy, the efficacy and safety of a combination of paclitaxcel given weekly and trastuzumab $(8 \mathrm{mg} / \mathrm{kg}$; every 3 weeks) were observed [35]. The ORR was 37\% (95\% CI 23-52\%) and the PFS and OS were 5.1 months (95\% CI 3.8-6.5 months) and 17.1 months (95\% CI 13.5-18.6 months) respectively. The grade 3 or higher adverse events reported in the trial were neutropenia (32.6\%), leukopenia (17.4\%), anemia (15.2\%), and hypoalbuminemia (8.7\%).

Lapatinib is a molecular targeted agent that targets HER2, similarly to trastuzumab, and also epidermal growth factor receptor (EGFR), unlike trastuzumab. The add-on effect of lapatinib with regard to capecitabine was verified in a phase III trial in patients with advanced breast cancer who had previously been treated with an anthracycline, a taxane, and trastuzumab [36]. The add-on effect of lapatinib with regard to paclitaxcel given weekly was evaluated in a phase III trial of second-line chemotherapy in HER2-positive patients with AGC [37]. Two hundred sixty-one patients were randomized into either a group that received lapatinib plus paclitaxcel weekly $(n=132)$ or a group that receivved paclitaxcel weekly $(n=129)$. The median OS was 11.0 months in the group that lapatinib plus paclitaxcel weekly and 8.9 months in the group that received paclitaxcel weekly (HR 0.84; 95\% CI 0.64-1.11; $P=0.1044$ ). In a subgroup analysis of OS by immunohistochemistry (IHC) status, lapatinib showed a tendency of an add-on effect among patients with IHC status $3+$. The results of this trial may have been affected by the fact that the percentage of enrolled patients with IHC stats 0 or $1+$ was as high as $35 \%$. It will be necessary to obtain data from a confirmatory trial in patients with IHC status $3+$ only.

Table 3 Ongoing trials for second-line patients in advanced gastric cancer-a summary of major trials

\begin{tabular}{|c|c|c|c|c|c|}
\hline Regimens & $\begin{array}{l}\text { Trial name/clinical trial } \\
\text { registration }\end{array}$ & Phase & Target & $\begin{array}{l}\text { No. of } \\
\text { patients }\end{array}$ & Locations \\
\hline Ramucirumab + nab-paclitaxel q1w & JapicCTI-153088 & II & VEGFR2 & 40 & Japan \\
\hline Ramucirumab + nab-paclitaxel q1w & NCT02317991 & II & VEGFR2 & 65 & USA \\
\hline Nimotuzumab + irinotecan & $\begin{array}{l}\text { ENRICH } \\
\text { NCT01813253 }\end{array}$ & III & EGFR & 400 & Asia \\
\hline BBI-608 + paclitaxel q1w & $\begin{array}{l}\text { BRIGHTER } \\
\text { NCT02178956 }\end{array}$ & III & STAT3 & 700 & Global \\
\hline Pembrolizumab & $\begin{array}{l}\text { KEYNOTE-061 } \\
\text { NCT02370498 }\end{array}$ & III & PD-1 & 720 & Global \\
\hline $\begin{array}{l}\text { Nivolumab }+ \text { oxaliplatin }+ \text { S- } 1 \text { or } \\
\text { capecitabine }\end{array}$ & NCT02746796 & II & PD-1 & 268 & Japan, Korea \\
\hline Nivolumab & NCT02267343 & III & PD-1 & 480 & $\begin{array}{l}\text { Japan, Korea, } \\
\text { Taiwan }\end{array}$ \\
\hline
\end{tabular}

EGFR epidermal growth factor receptor, $P D-1$ programmed cell death $1, q 1 w$ every week, STAT3 signal transducer and activator of transcription $3, V E G F R 2$ vascular endothelial growth factor receptor 2 
Ado-trastuzumab emtansine (T-DM1), the other novel anti-HER2 monoclonal antibody, is a molecular targeted agent consisting of trastuzumab linked to emtansine (DM1), a microtubule inhibitor. The efficacy and safety of second-line chemotherapy with T-DM1 were compared with those of a taxane in HER2-positive patients with AGC or advanced esophagogastric junction (EGJ) cancer in a phase II/III trial [38]. On the basis of advice given by the independent data monitoring committee after completion of the phase II part of the trial, the recommended dose of T-DM1 for the phase III part of the trial was determined to be $2.4 \mathrm{mg} / \mathrm{kg}$, administered once weekly. Three hundred forty-five patients were randomized into either a group that received T-DM1 at $2.4 \mathrm{mg} / \mathrm{kg}(n=228)$ or a taxane group $(n=117)$ in 28 countries. The taxane group consisted of 69 patients in the cohort hat received docetaxel every 3 weeks and 42 patients in cohort that received paclitaxcel every week. The median OS was 7.9 months in the T-DM1 group and 8.6 months in the taxane group (HR 1.15; $95 \%$ CI $0.87-1.51 ; P=0.8589$ ), failing to show any superior clinical benefits of T-DM1 over taxane, a standard treatment, in terms of efficacy. On the basis of the results of this trial, the development of T-DM1 for the treatment of gastric cancer was discontinued.

\section{Anti-VEGF antibody}

Bevacizumab, a molecular targeted agent binding specifically to vascular endothelial growth factor (VEGF), has demonstrated its efficacy against colorectal cancer, nonsmall-cell lung cancer, breast cancer, ovarian cancer, cervical cancer, and malignant glioma. The agent is also being tested for the treatment of gastric cancer. A phase III trial (AVAGAST) of first-line chemotherapy for patients with AGC or advanced EGJ cancer was conducted to investigate the add-on effect of bevacizumab with regard to capecitabine plus cisplatin (XP) or 5-FU plus cisplatin (FP) [39]. The median OS was 12.1 months in the XP/FP plus bevacizumab group and 10.1 months in the XP/FP plus placebo group (HR 0.87; 95\% CI 0.73-1.03; $P=0.1002$ ), showing no significant prolongation of OS when bevacizumab was added to the therapy. Of the enrolled patients in the respective regions, the following percentages of patients received second-line chemotherapy: 66\% in the Asia-Pacific region (OS 12.1 months), 31\% in the European region (OS 8.6 months), and $21 \%$ in the pan-American region (OS 6.8 months). These OS data by region suggest the possibility of second-line chemotherapy being a factor for the prolongation of survival.

Ramucirumab is a recombinant human monoclonal immunoglobulin G1 antibody against human VEGF receptor 2 (VEGFR2), which plays an important role in angiogenesis involved in cancer growth and metastases. Ramucirumab binds specifically to the extracellular domain of VEGFR2 and inhibits the interaction of VEGFR2 with its ligands (not only VEGF-A, but also VEGF-C, and VEGF-D), and thereby inhibits signaling and angiogenesis of cancer, exerting an antitumor effect. Two phase III trials (REGARD and RAINBOW) of second-line therapy for patients with AGC were conducted [40, 41]. In the REGARD trial, the efficacy and safety of second-line therapy with ramucirumab were evaluated in patients with AGC or advanced EGJ cancer who had received first-line chemotherapy [40]. Three hundred fifty-five patients (238 in the ramucirumab group and 117 in the placebo group) were enrolled in the trial in 29 countries (Japan did not participate). The median OS was 5.2 months in the ramucirumab group and 3.8 months in the placebo group (HR $0.776 ; 95 \%$ CI $0.603-0.998 ; P=0.047)$, demonstrating the superiority of ramucirumab over placebo. The incidence of all grades adverse events was $94 \%$ in the ramucirumab group, which was similar to that in the placebo group (88\%). No grade 3 or higher adverse events with an incidence of more than $10 \%$ were reported in the ramucirumab group. The incidence of hypertension, a VEGFantibody-specific event, was $16 \%$ in the ramucirumab group, which was higher than that in the placebo group $(8 \%)$. Ramucirumab was not associated with increased rates of fatigue, emesis, or other notable toxic effects. Ramucirumab, anti-VEGFR2 antibody, is the first molecular targeted agent that has demonstrated efficacy of second-line therapy in patients with AGC.

The add-on effect of ramucirumab with regard to paclitaxcel, a standard chemotherapy agent used as secondline treatment for AGC, was observed in the RAINBOW trial [41]. Six hundred sixty-five patients were enrolled in the trial in 27 countries. Of these 665 patients, 330 patients were randomized into a group that received paclitaxcel weekly plus ramucirumab and 335 patients were randomized into a group that received paclitaxcel weekly. The median follow-up time was 7.9 months. The median OS was 9.6 months in the group that received paclitaxcel weekly plus ramucirumab group and 7.4 months in the group that received paclitaxcel weekly (HR 0.807 ; $95 \%$ CI $0.678-0.962 ; P=0.017)$, demonstrating the add-on effect of ramucirumab. The PFS was 4.4 months in the group that received paclitaxcel weekly plus ramucirumab and 2.9 months in the group that received paclitaxcel weekly (HR 0.635; 95\% CI 0.536-0.752; $P<0.0001$ ), demonstrating that ramucirumab had an add-on effect for PFS similar to that seen for OS. The results of subgroup analyses conducted to compare Japanese and Western patients in the RAINBOW trial were also reported [42]. In the 68 Japanese patients in the group that received paclitaxcel weekly plus ramucirumab and in the 72 Japanese patients 
in the group that received paclitaxcel weekly, the median OS was 11.4 months and 11.5 months respectively (HR $0.880 ; P=0.5113$ ) and the median PFS was 5.6 months and 2.8 months respectively (HR 0.503; $P=0.0002$ ). In the 198 Western patients in the group that received paclitaxcel weekly plus ramucirumab and the 200 Western patients in the group that received paclitaxcel weekly, the median OS was 8.6 months and 5.9 months respectively (HR 0.726; $P=0.0050$ ) and the median PFS was 4.2 months and 2.8 months respectively (HR, 0.631; $P \leq 0.0001)$. These results showed that the Japanese subgroup achieved an improvement in PFS, though not in OS. Ramucirumab therapy and weekly paclitaxcel therapy plus ramucirumab therapy are recommended as standard second-line chemotherapy for AGC in the National Comprehensive Cancer Network clinical practice guidelines [43], the European Society for Medical Oncology clinical practice guidelines [44], and the Japanese gastric cancer treatment guidelines [45]. Phase II trials of combination therapy with nab-paclitaxcel given weekly and ramucirumab (JapicCTI-153088, NCT02317991) are being conducted in Japan and the USA and are expected to contribute to the development of more treatment approaches.

\section{Mammalian target of rapamycin inhibitor}

Mammalian target of rapamycin, located in the downstream part of the phosphatidylinositol 3-kinase/Akt signaling pathway regulates cell proliferation and cell death. Everolimus is an orally administered mammalian target of rapamycin inhibitor, and its efficacy has been confirmed in the treatment of various types of cancer. The efficacy of everolimus as second- and third-line chemotherapy for AGC was evaluated in the GRANITE- 1 trial. The median OS was 5.4 months in the everolimus group and 4.3 months in the placebo group (HR 0.90; 95\% CI $0.75-1.08 ; P=0.124)$, failing to show the efficacy of everolimus [46]. After completion of enrollment in the GRANITE-1 trial, the GRANITE-2 trial (NCT01248403) was started to verify the add-on effect of everolimus with regard to weekly paclitaxcel therapy. However, since the efficacy of everolimus could not be verified in the GRANITE-1 trial, the GRANITE-2 trial was also terminated and the development of everolimus for the treatment of gastric cancer was discontinued.

\section{Poly(ADP-ribose) polymerase inhibitor}

In the BRCA1/BRCA2 gene involved in the homologous recombination pathway for DNA repair, loss of function associated with genetic mutation is known in breast cancer and ovarian cancer. Poly(ADP-ribose) polymerase (PARP) is involved in base excision repair, a DNA modification. Cells with the BRCA1/BRCA2 gene mutation cannot repair DNA damage caused by PARP inhibition and this eventually result in cell death. Olaparib, an orally administered PARP inhibitor, acts specifically on cells with DNA repair pathway defects and induces cell death. It is the first PARP inhibitor to be approved for the indication of BRCA gene mutation positive advanced ovarian cancer in the USA and the European Union and is also effective in the treatment of BRCApositive prostate cancer [47, 48]. Preclinical data suggested that olaparib, a PARP inhibitor, is effective for treating gastric cancer patients with a low ataxia telangiectasia mutated (ATM) protein level [49]. A study to evaluate add-on effect of olaparib with regard to weekly paclitaxcel therapy as second-line chemotherapy for patients with AGC was conducted in Korea [50]. In this study, 124 patients were treated with either paclitaxcel weekly plus olaparib or paclitaxcel weekly plus placebo. The median duration of follow-up for the overall population was 8.4 months (0.3-26.2 months). The PFS, the primary end point, was 3.91 months with olaparib versus 3.55 months with weekly paclitaxcel therapy plus placebo (HR $0.80 ; 80 \%$ CI $0.62-1.03 ; P=0.131$ ). The OS, the secondary end point, was 13.1 months with olaparib versus 8.3 months with weekly paclitaxcel therapy plus placebo (HR $0.56 ; 95 \%$ CI $0.35-0.87$; $P=0.010 ; 80 \%$ CI $0.41-0.75 ; P=0.005)$. In the low ATM expression population, the OS in the group that received paclitaxcel weekly plus olaparib was not reached because of lack of events and in the group that received paclitaxcel weekly plus placebo it was 8.2 months (HR $0.35 ; 80 \%$ CI $0.22-0.56 ; P=0.002$ ). On the basis of these results, a phase III trial (GOLD) was conducted in four Asian countries [51]. The experimental group received paclitaxcel weekly plus olaparib $(100 \mathrm{mg}$ tablet twice daily) and the control group received paclitaxcel weekly plus placebo. This trial used co-primary end points to evaluate the OS in all patients and ATM protein negative patients. The median OS in all patients was 8.8 months in the group that received paclitaxcel weekly plus olaparib and 6.9 months in the group that received paclitaxcel weekly plus placebo (HR 0.79; 97.5\% CI 0.63-1.00; $P=0.0262$ ). The OS in ATM protein-negative patients was 12.0 months in the group that received paclitaxcel weekly plus olaparib group and 10.0 months in the group that received paclitaxcel weekly plus placebo (HR $0.73 ; 97.5 \%$ CI $0.40-1.34$; $P=0.2458$ ), showing no statistically significant differences between these patient populations. Because of these results, it has become difficult to pursue the development of olaparib for the treatment of AGC. 


\section{EGFR inhibitor}

To develop an anti-EGFR antibody for the treatment of AGC, two phase III trials (REAL-3 and EXPAND) were conducted. Although the REAL-3 trial was conducted to verify the add-on effect of panitumumab with regard to a combination of epirubicin, oxaliplatin, and capecitabine, and the EXPAND trial was conducted to verify the add-on effect of cetuximab with respect to XP, neither study could verify that anti-EGFR antibody agents had any add-on effects $[52,53]$.

Nimotuzumab is a humanized immunoglobulin G1 antibody against EGFR. The effect of a combination of irinotecan and nimotuzumab was compared with that of single-agent irinotecan as second-line chemotherapy for patients with AGC in a randomized phase II trial [54]. The PFS was 73.0 days in the irinotecan group and 85.0 days in the irinotecan plus nimotuzumab group (HR 0.860; $P=0.5668$ ), and the OS was 250.5 days and 232.0 days respectively (HR 0.994; $P=0.9778$ ), showing no statistically significant differences between the two groups. However, in a subgroup analysis in patients who had the highest EGFR expression (2+/3+), the PFS was 59.0 days in the irinotecan group and 118.5 days in the irinotecan plus nimotuzumab group (HR 0.341; $P=0.1293$ ), and the OS was 229.5 days and 358.5 days respectively (HR 0.369; $P=0.0944)$. On the basis of this subgroup analysis in the randomized phase II trial, a phase III trial (NCT01813253) of second-line chemotherapy is currently under way in EGFR $2+/ 3+$ patients with AGC.

\section{Signal transducer and activator of transcription 3 inhibitor}

Signal transducer and activator of transcription 3 (STAT3), a transcription factor, is constantly activated in many cancer cells, and the inhibition of STAT3 activation is known to inhibit the growth of cancer cell lines $[55,56]$. BBI-608, an orally administered anticancer agent against STAT3 that is designed to inhibit cancer stem cell pathways, is being developed for the treatment of various types of cancer. In a phase I trial of a combination of weekly paclitaxcel and BBI-608 in patients with solid cancer, five patients with gastric or EGJ cancer were enrolled. Tumor reduction was observed in three of the five patients $(45 \%, 48 \%$, and $24 \%$ respectively), and stable disease was maintained in two patients for 5 months or more [57]. In a phase I trial in six Japanese patients with gastric or EGJ cancer, the response rate was $33.3 \%$, and one of the six patients was reported to maintain the response for 7.5 months or more [58]. A global phase III trial (NCT02178956) is under way in patients with AGC to compare weekly paclitaxcel plus BBI-608 with weekly paclitaxcel as second-line chemotherapy.

\section{Immune checkpoint inhibitors}

The programmed cell death 1 (PD-1)/programmed death ligand 1 (PD-L1) pathway, an immune checkpoint, is involved in the regulation of cellular immune function. In recent years, anti-PD-1 antibodies and anti-PD-L1 antibodies, inhibitors of this pathway, have been actively developed. In particular, anti-PD-1 antibodies demonstrated their high efficacy compared with conventional cytotoxic antitumor agents or ipilimumab, an anti-cytotoxic T-lymphocyte-associated protein 4 antibody approved in the USA in 2011, and are widely used in clinical practice. Currently, pembrolizumab and nivolumab are being developed as anti-PD-1 antibodies for the treatment of AGC (Table 3). As of November 2016, pembrolizumab, a PD-1 antibody, was approved by the FDA for treatment of melanoma, non-small-cell lung cancer and head and neck cancer, and nivolumab was also approved by the FDA for treatment of non-small-cell lung cancer, melanoma, renal cell carcinoma and Hodgkin lymphoma.

Pembrolizumab was evaluated in a phase $\mathrm{Ib}$ trial (KEYNOTE-012) for heavily pretreated patients with PD-L1-positive AGC. Of 162 patients screened, 65 patients (40\%) were found to be positive for PD-L1. In the trial, 39 of the 65 patients were enrolled and the overall response rate was $22 \%$ ( 8 of the 36 patients achieved response). The median PFS and the median OS were 1.9 months $(95 \%$ CI 1.8-3.5 months) and 11.4 months (95\% CI 5.7 months to not reacted) respectively [59]. A phase III trial (KEYNOTE-061) of second-line chemotherapy for PD-L1-positive patients is being conducted to compare paclitaxcel given weekly and pembrolizumab (NCT02370498). If the superiority of pembrolizumab over paclitaxcel given weekly is demonstrated in the trial, basic second-line chemotherapy for PD-L1-positive patients with AGC could be replaced by pembrolizumab and standard treatments could undergo a major change. Given that its clinical positioning relative to weekly paclitaxcel plus ramucirumab, the current standard chemotherapy, is still unknown, further investigation of pembrolizumab will be needed.

A phase I/II trial (CheckMate-032) evaluated the efficacy and safety of nivolumab as a single agent and in combination with ipilimumab in patients with AGC or advanced EGJ cancer [60]. Fifty-nine patients received treatment with nivolumab, and the overall response rate was $14 \%$ ( 1 complete response, 7 partial responses) and the median OS was 5.0 months (95\% CI 3.4-12.4 months). 
The trial enrolled patients irrespective of their PD-L1 status. The overall response rate was $27 \%$ when the cutoff for PD-L1 positivity was set at $1 \%$ or more and $33 \%$ when the cutoff was set at $5 \%$ or more. Nivolumab is being evaluated in a phase II trial (NCT02746796) as first-line immunotherapy and in a phase III trial (NCT02267343) as third-line immunotherapy. However, no clinical trials of nivolumab as second-line immunotherapy have been conducted.

Avelumab, an anti-PD-L1 antibody, has also been developed for the treatment of patients with AGC. Although the JAVELIN Gastric 100 trial (NCT02625610) of first-line immunotherapy and the JAVLIN Gastric 300 trial (NCT02625623) of third-line immunotherapy are being conducted, no clinical trials of avelumab as secondline immunotherapy have been conducted.

\section{Future perspectives}

Second-line chemotherapy should be offered to patients who are maintaining good performance status because its superiority over BSC has been reported on the basis of data from a number of phase III trials [30-32]. Whereas firstline treatment strategies for patients with AGC differ depending on the country, weekly paclitaxcel in combination with treatment with ramucirumab, a molecular targeted agent, has been established as standard second-line chemotherapy on the basis of data from past clinical developments. Although the proportion of patients receiving second-line chemotherapy differs depending on the country, clinical developments for second-line and laterline chemotherapy should be continued to achieve further prolongation of survival.

It is also important to develop cytotoxic anticancer agents. Currently, first-line chemotherapy with TAS-118 (SOLAR; NCT02322593), IMAB362 (FAST; NCT01630083), and intraperitoneally administered paclitaxcel (Phoenix-GC; UMIN000005930) and third-line chemotherapy with TAS-102 (TAGS; NCT02500043) are being evaluated in clinical trials. They may become promising second-line chemotherapeutic agents depending on the results of the trials. Similarly, immune checkpoint inhibitors are also developed mainly as first-line or thirdline therapy. Depending on the results of the KEYNOTE061 trial, however, standard second-line therapy may undergo a major change, and other immune checkpoint inhibitors may be more actively developed.

\section{Compliance with ethical standards}

This article does not contain any studies with human or animal subjects performed by the author. Also, in all studies cited it was declared that informed consent was obtained from all patients for their being included in the studies.

Conflict of interest Daisuke Takahari has received grants from Taiho Pharmaceutical Co. Ltd., Yakult Honsha, and Eli Lilly Japan K.K.

\section{References}

1. Torre LA, Bray F, Siegel RL, Ferlay J, Lortet-Tieulent J, Jemal A, et al. Global cancer statistics, 2012. CA Cancer J Clin. 2015;65:87-108.

2. Murad AM, Santiago FF, Petroianu A, Rocha PR, Rodrigues MA, Rausch M. Modified therapy with 5-fluorouracil, doxorubicin, and methotrexate in advanced gastric cancer. Cancer (Phila). 1993;72:37-41.

3. Glimelius B, EkstrÖm K, Hoffman K, Graf W, SjÖdén PO, Haglund U, et al. Randomized comparison between chemotherapy plus best supportive care with best supportive care in advanced gastric cancer. Ann Oncol. 1997;8:163-8.

4. Pyrhönen S, Kuitunen T, Nyandoto P, Kouri M. Randomised comparison of fluorouracil, epidoxorubicin and methotrexate (FEMTX) plus supportive care with supportive care alone in patients with non-resectable gastric cancer. $\mathrm{Br} \mathrm{J}$ Cancer. 1995;71:587-91.

5. Wagner AD, Grothe W, Haerting J, Kleber G, Grothey A, Fleig WE. Chemotherapy in advanced gastric cancer: a systematic review and meta-analysis based on aggregate data. J Clin Oncol. 2006;24:2903-9.

6. Kim NK, Park YS, Heo DS, Suh C, Kim SY, Park KC, et al. A phase III randomized study of 5-fluorouracil and cisplatin versus 5-fluorouracil, doxorubicin, and mitomycin $\mathrm{C}$ versus 5-fluorouracil alone in the treatment of advanced gastric cancer. Cancer. 1993;71:3813-8.

7. Vanhoefer U, Rougier P, Wilke H, Ducreux MP, Lacave AJ, Van Cutsem E, et al. Final results of a randomized phase III trial of sequential high-dose methotrexate, fluorouracil, and doxorubicin versus etoposide, leucovorin, and fluorouracil versus infusional fluorouracil and cisplatin in advanced gastric cancer: a trial of the European Organization for Research and Treatment of Cancer Gastrointestinal Tract Cancer Cooperative Group. J Clin Oncol. 2000;18:2648-57.

8. Ohtsu A, Shimada Y, Shirao K, Boku N, Hyodo I, Saito H, et al. Randomized phase III trial of fluorouracil alone versus fluorouracil plus cisplatin versus uracil and tegafur plus mitomycin in patients with unresectable, advanced gastric cancer: the Japan Clinical Oncology Group study (JCOG9205). J Clin Oncol. 2003;21:54-9.

9. Van Cutsem E, Moiseyenko VM, Tjulandin S, Majlis A, Constenla M, Boni C, et al. Phase III study of docetaxel and cisplatin plus fluorouracil compared with cisplatin and fluorouracil as firstline therapy for advanced gastric cancer: a report of the V325 Study Group. J Clin Oncol. 2006;24:4991-7.

10. Cunningham D, Starling N, Rao S, Iveson T, Nicolson M, Coxon $\mathrm{F}$, et al. Capecitabine and oxaliplatin for advanced esophagogastric cancer. N Engl J Med. 2008;358:36-46.

11. Boku N, Yamamoto S, Fukuda H, Shirao K, Doi T, Sawaki A, et al. Fluorouracil versus combination of irinotecan plus cisplatin versus S-1 in metastatic gastric cancer: a randomised phase 3 study. Lancet Oncol. 2009;10:1063-9.

12. Koizumi W, Narahara H, Hara T, Takagane A, Akiya T, Takagi M, et al. S-1 plus cisplatin versus S-1 alone for first-line treatment of advanced gastric cancer (SPIRITS trial): a phase III trial. Lancet Oncol. 2008;9:215-21. 
13. Takashima A, Boku N, Kato K, Nakamura K, Mizusawa J, Fukuda $\mathrm{H}$, et al. Survival prolongation after treatment failure of first-line chemotherapy in patients with advanced gastric cancer: combined analysis of the Japan Clinical Oncology Group trials JCOG9205 and JCOG9912. Gastric Cancer. 2014;17:522-8.

14. Graziano F, Catalano V, Baldelli AM, Giordani P, Testa E, Lai V, et al. A phase II study of weekly docetaxel as salvage chemotherapy for advanced gastric cancer. Ann Oncol. 2000;11:1263-6.

15. Assersohn L, Brown G, Cunningham D, Ward C, Oates J, Waters JS, et al. Phase II study of irinotecan and 5-fluorouracil/leucovorin in patients with primary refractory or relapsed advanced oesophageal and gastric carcinoma. Ann Oncol. 2004;15:64-9.

16. Giuliani F, Molica S, Maiello E, Battaglia C, Gebbia V, Di Bisceglie M, et al. Irinotecan (CPT-11) and mitomycin-C (MMC) as second-line therapy in advanced gastric cancer: a phase II study of the Gruppo Oncologico dell' Italia Meridionale (prot. 2106). Am J Clin Oncol. 2005;28:581-5.

17. Yamada Y, Shirao K, Ohtsu A, Boku N, Hyodo I, Saitoh H, et al. Phase II trial of paclitaxel by three-hour infusion for advanced gastric cancer with short premedication for prophylaxis against paclitaxel-associated hypersensitivity reactions. Ann Oncol. 2001;12:1133-7.

18. Yamaguchi K, Tada M, Horikoshi N, Otani T, Takiuchi H, Saitoh S. Phase II study of paclitaxel with 3-h infusion in patients with advanced gastric cancer. Gastric Cancer. 2002;5:90-5.

19. Kodera Y, Ito S, Mochizuki Y, Fujitake S, Koshikawa K, Kanyama Y, et al. A phase II study of weekly paclitaxel as second-line chemotherapy for advanced gastric cancer (CCOG0302 study). Anticancer Res. 2007;27:2667-71.

20. Matsuda G, Kunisaki C, Makino H, Fukahori M, Kimura J, Sato $\mathrm{T}$, et al. Phase II study of weekly paclitaxel as a second-line treatment for S-1-refractory advanced gastric cancer. Anticancer Res. 2009;29:2863-7.

21. Im CK, Rha SY, Jeung HC, Jeong J, Lee SH, Noh SH, et al. A phase II feasibility study of weekly paclitaxel in heavily pretreated advanced gastric cancer patients with poor performance status. Oncology. 2009;77:349-57.

22. Hamaguchi T, Shirao K, Ohtsu A, Hyodo I, Arai Y, Takiuchi H, et al. A phase II study of biweekly mitomycin $\mathrm{C}$ and irinotecan combination therapy in patients with fluoropyrimidine-resistant advanced gastric cancer: a report from the Gastrointestinal Oncology Group of the Japan Clinical Oncology Group (JCOG0109-DI trial). Gastric Cancer. 2011;14:226-33.

23. Shitara K, Yuki S, Takahari D, Nakamura M, Kondo C, Tsuda T, et al. Randomised phase II study comparing dose-escalated weekly paclitaxel vs standard-dose weekly paclitaxel for patients with previously treated advanced gastric cancer. $\mathrm{Br} \mathrm{J}$ Cancer. 2014;110:271-7.

24. Nishina T, Boku N, Gotoh M, Shimada Y, Hamamoto Y, Yasui $\mathrm{H}$, et al. Randomized phase II study of second-line chemotherapy with the best available 5-fluorouracil regimen versus weekly administration of paclitaxel in far advanced gastric cancer with severe peritoneal metastases refractory to 5-fluorouracil-containing regimens (JCOG0407). Gastric Cancer. 2016;19:902-10.

25. Higuchi K, Tanabe S, Shimada K, Hosaka H, Sasaki E, Nakayama $\mathrm{N}$, et al. Biweekly irinotecan plus cisplatin versus irinotecan alone as second-line treatment for advanced gastric cancer: a randomised phase III trial (TCOG GI-0801/BIRIP trial). Eur J Cancer. 2014;50:1437-45.

26. Nishikawa K, Fujitani K, Inagaki H, Akamaru Y, Tokunaga S, Takagi $\mathrm{M}$, et al. Randomised phase III trial of second-line irinotecan plus cisplatin versus irinotecan alone in patients with advanced gastric cancer refractory to S-1 monotherapy: tRICS trial. Eur J Cancer. 2015;51:808-16.
27. Tanabe K, Fujii M, Nishikawa K, Kunisaki C, Tsuji A, Matsuhashi N, et al. Phase II/III study of second-line chemotherapy comparing irinotecan-alone with S-1 plus irinotecan in advanced gastric cancer refractory to first-line treatment with S-1 (JACCRO GC-05). Ann Oncol. 2015;26:1916-22.

28. Thuss-Patience PC, Kretzschmar A, Bichev D, Deist T, Hinke A, Breithaupt K, et al. Survival advantage for irinotecan versus best supportive care as second-line chemotherapy in gastric cancer: a randomised phase III study of the Arbeitsgemeinschaft Internistische Onkologie (AIO). Eur J Cancer. 2011;47:2306-14.

29. Kang JH, Lee SI, Lim DH, Park KW, Oh SY, Kwon HC, et al. Salvage chemotherapy for pretreated gastric cancer: a randomized phase III trial comparing chemotherapy plus best supportive care with best supportive care alone. J Clin Oncol. 2012;30:1513-8.

30. Ford HE, Marshall A, Bridgewater JA, Janowitz T, Coxon FY, Wadsley J, et al. Docetaxel versus active symptom control for refractory oesophagogastric adenocarcinoma (COUGAR-02): an open-label, phase 3 randomised controlled trial. Lancet Oncol. 2014;15(1):78-86.

31. Hironaka S, Ueda S, Yasui H, Nishina T, Tsuda M, Tsumura T, et al. Randomized, open-label, phase III study comparing irinotecan with paclitaxel in patients with advanced gastric cancer without severe peritoneal metastasis after failure of prior combination chemotherapy using fluoropyrimidine plus platinum: WJOG 4007 trial. J Clin Oncol. 2013;31:4438-44.

32. Sasaki Y, Nishina T, Yasui H, Goto M, Muro K, Tsuji A, et al. Phase II trial of nanoparticle albumin-bound paclitaxel as secondline chemotherapy for unresectable or recurrent gastric cancer. Cancer Sci. 2014;105:812-7.

33. Shitara K, Takashima A, Fujitani K, Koeda K, Hara H, Nakayama N, et al. Nab-paclitaxel versus solvent-based paclitaxel in patients with previously treated advanced gastric cancer (ABSOLUTE): an open-label, randomised, non-inferiority, phase 3 trial. Lancet Gastroenterol Hepatol. 2017. doi:10.1016/S24681253(16)30219-9.

34. Bang YJ, Van Cutsem E, Feyereislova A, Chung HC, Shen L, Sawaki A, et al. Trastuzumab in combination with chemotherapy versus chemotherapy alone for treatment of HER2-positive advanced gastric or gastrooesophageal junction cancer (ToGA): a phase 3, open-label, randomised controlled trial. Lancet. 2010;376:687-97.

35. Nishikawa K, Takahashi T, Takaishi H, Miki A, Noshiro H, Yoshikawa T, et al. Phase II study of the effectiveness and safety of trastuzumab and paclitaxel for taxane- and trastuzumab-naïve patients with HER2-positive, previously treated, advanced gastric cancer (JFMC45-1102). Int J Cancer. 2016. doi:10.1002/ijc. 30383.

36. Geyer CE, Forster J, Lindquist D, Chan S, Romieu CG, Pienkowski T, et al. Lapatinib plus capecitabine for HER2-positive advanced breast cancer. N Engl J Med. 2006;355:2733-43.

37. Satoh T, Xu RH, Chung HC, Sun GP, Doi T, Xu JM, et al. Lapatinib plus paclitaxel versus paclitaxel alone in the secondline treatment of HER2-amplified advanced gastric cancer in Asian populations: TyTAN-a randomized, phase III study. J Clin Oncol. 2014;32:2039-49.

38. Kang YK, Shah MA, Ohtsu A, Van Cutsem E, Ajani JA, van der Horst $\mathrm{F}$, et al. A randomized, open-label, multicenter, adaptive phase $2 / 3$ study of trastuzumab emtansine (T-DM1) versus a taxane (TAX) in patients (pts) with previously treated HER2positive locally advanced or metastatic gastric/gastroesophageal junction adenocarcinoma (LA/MGC/GEJC). J Clin Oncol. 2016;34(4 Suppl 4):5.

39. Ohtsu A, Shah MA, Van Cutsem E, Rha SY, Sawaki A, Park SR, et al. Bevacizumab in combination with chemotherapy as firstline therapy in advanced gastric cancer: a randomized, double- 
blind, placebo-controlled phase III study. J Clin Oncol. 2011;29:3968-76.

40. Fuchs CS, Tomasek J, Yong CJ, Dumitru F, Passalacqua R, Goswami C, et al. Ramucirumab monotherapy for previously treated advanced gastric or gastro-oesophageal junction adenocarcinoma (REGARD): an international, randomised, multicentre, placebo-controlled, phase 3 trial. Lancet. 2014;383:31-9.

41. Wilke H, Muro K, Van Cutsem E, Oh SC, Bodoky G, Shimada Y, et al. Ramucirumab plus paclitaxel versus placebo plus paclitaxel in patients with previously treated advanced gastric or gastrooesophageal junction adenocarcinoma (RAINBOW): a doubleblind, randomised phase 3 trial. Lancet Oncol. 2014;15:1224-35.

42. Shitara K, Muro K, Shimada Y, Hironaka S, Sugimoto N, Komatsu Y, et al. Subgroup analyses of the safety and efficacy of ramucirumab in Japanese and Western patients in RAINBOW: a randomized clinical trial in second-line treatment of gastric cancer. Gastric Cancer. 2016;19:927-38.

43. National Comprehensive Cancer Network. NCCN guidelines for gastric cancer 2016 (ver. 3). https://www.nccn.org/professionals/ physician_gls/pdf/gastric.pdf. Accessed 3 Mar 2017

44. Smyth EC, Verheij M, Allum W, Cunningham D, Cervantes A, Arnold D, et al. Gastric cancer: ESMO clinical practice guidelines for diagnosis, treatment and follow-up. Ann Oncol. 2016;27(Suppl 5):v38-49.

45. Japanese Gastric Cancer Association. Japanese gastric cancer treatment guidelines 2014 (ver. 4). Gastric Cancer. 2017;20:1-19.

46. Ohtsu A, Ajani JA, Bai YX, Bang YJ, Chung HC, Pan HM, et al. Everolimus for previously treated advanced gastric cancer: results of the randomized, double-blind, phase III GRANITE-1 study. J Clin Oncol. 2013;31:3935-43.

47. Ledermann J, Harter P, Gourley C, Friedlander M, Vergote I, Rustin $\mathrm{G}$, et al. Olaparib maintenance therapy in platinum-sensitive relapsed ovarian cancer. N Engl J Med. 2012;366:1382-92.

48. Mateo J, Carreira S, Sandhu S, Miranda S, Mossop H, PerezLopez R, et al. DNA-repair defects and olaparib in metastatic prostate cancer. N Engl J Med. 2015;373:1697-708.

49. Kubota E, Williamson CT, Ye R, et al. Low ATM protein expression and depletion of p53 correlates with olaparib sensitivity in gastric cancer cell lines. Cell Cycle. 2014;13:2129-37.

50. Bang YJ, Im SA, Lee KW, Cho JY, Song EK, Lee KH, et al. Randomized, double-blind phase II trial with prospective classification by ATM protein level to evaluate the efficacy and tolerability of olaparib plus paclitaxel in patients with recurrent or metastatic gastric cancer. J Clin Oncol. 2015;33:3858-65.
51. Bang YJ, Boku N, Chin K, Lee KW, Park SH, Qin S, et al. Olaparib in combination with paclitaxel in patients with advanced gastric cancer who have progressed following first-line therapy: phase III GOLD study. Ann Oncol. 2016:27(6):1-36.

52. Waddell T, Chau I, Cunningham D, Gonzalez D, Okines AF, Okines $\mathrm{C}$, et al. Epirubicin, oxaliplatin, and capecitabine with or without panitumumab for patients with previously untreated advanced oesophagogastric cancer (REAL3): a randomised, open-label phase 3 trial. Lancet Oncol. 2013;14:481-9.

53. Lordick F, Kang YK, Chung HC, Salman P, Oh SC, Bodoky G, et al. Capecitabine and cisplatin with or without cetuximab for patients with previously untreated advanced gastric cancer (EXPAND): a randomised, open-label phase 3 trial. Lancet Oncol. 2013;14:490-9.

54. Satoh T, Lee KH, Rha SY, Sasaki Y, Park SH, Komatsu Y, et al. Randomized phase II trial of nimotuzumab plus irinotecan versus irinotecan alone as second-line therapy for patients with advanced gastric cancer. Gastric Cancer. 2015;18:824-32.

55. Bromberg JF, Wrzeszczynska MH, Devgan G, Zhao Y, Pestell RG, Albanese C, et al. The JAK-STAT pathway: summary of initial studies and recent advances. Stat 3 as an oncogene. Cell. 1999;98:295-303.

56. Bromberg JF, Horvath CM, Besser D, Lathem WW, Darnell JE Jr. Stat 3 activation is required for cellular transformation by v-src. Mol Cell Biol. 1998;18:2553-8.

57. Hitron M, Stephenson J, Chi KN, Edenfield WJ, Leggett D, Li Y, et al. A phase Ib study of the cancer stem cell inhibitor BBI608 administered with paclitaxel in pts with advanced malignancies. J Clin Oncol. 2014;32(15 Suppl):2530.

58. Shitara K, Kuboki Y, Nakamura Y, Doi A, Harada K, Kawazoe A, et al. A phase I study of BBI608, a cancer stemness inhibitor, administered with paclitaxel (PTX) as combination therapy (Rx) for pretreated unresectable or recurrent gastric cancer. J Clin Oncol. 2015;33(15 Suppl):3023.

59. Muro K, Chung HC, Shankaran V, Geva R, Catenacci D, Gupta $\mathrm{S}$, et al. Pembrolizumab for patients with PD-L1-positive advanced gastric cancer (KEYNOTE-012): a multicentre, openlabel, phase 1b trial. Lancet Oncol. 2016;17:717-26.

60. Le DT, Bendell JC, Calvo E, Kim JW, Ascierto PA, Sharma P, et al. Safety and activity of nivolumab monotherapy in advanced and metastatic $(\mathrm{A} / \mathrm{M})$ gastric or gastroesophageal junction cancer (GC/GEC): results from the CheckMate-032 study. J Clin Oncol. 2016;34(4 Suppl):6. 